\title{
Ground State of a Heisenberg Chain with Next-Nearest-Neighbor Bond Alternation
}

\author{
Luca Capriotti, ${ }^{1}$ Federico Becca, ${ }^{2,3}$ Sandro Sorella, ${ }^{3}$ and Alberto Parola,${ }^{4}$ \\ ${ }^{1}$ Kavli Institute for Theoretical Physics and Department of Physics, \\ University of California, Santa Barbara CA 93106-4030 \\ 2 Institut de Physique Théorique, Université de Lausanne, CH-1015 Lausanne, Switzerland \\ 3 INFM-DEMOCRITOS, National Simulation Centre, and SISSA, I-34014 Trieste, Italy \\ 4 INFM and Dipartimento di Scienze, Università dell'Insubria, I-22100 Como, Italy
}

(Dated: November 16, 2018)

\begin{abstract}
We investigate the ground-state properties of the spin-half $J_{1}-J_{2}$ Heisenberg chain with a nextnearest-neighbor spin-Peierls dimerization using conformal field theory and Lanczos exact diagonalizations. In agreement with the results of a recent bosonization analysis by Sarkar and Sen, we find that for small frustration $\left(J_{2} / J_{1}\right)$ the system is in a Luttinger spin-fluid phase, with gapless excitations, and a finite spin-wave velocity. In the regime of strong frustration the ground state is spontaneously dimerized and the bond alternation reduces the triplet gap, leading to a slight enhancement of the critical point separating the Luttinger phase from the gapped one. An accurate determination of the phase boundary is obtained numerically from the study of the excitation spectrum.
\end{abstract}

PACS numbers: 75.10.Jm,71.10.Hf,75.10.-b

Asymmetric spin-half ladders have recently attracted much theoretical interest 1.2 .3 .4 .5 due to possible experimental realizations in delafossite cuprates such as $\mathrm{YCuO}_{2.5}$ (Ref. 6) and the unusual physical effects that the asymmetry in the leg exchanges could introduce both in the ground-state correlations and in the properties of the excitation spectrum. The simplest and most transparent Hamiltonian representation of these systems can be given in terms of frustrated $\left(J_{1}-J_{2}\right)$ spin-half Heisenberg chains with a spin-Peierls dimerization in the nextnearest-neighbor (n.n.n.) interaction, namely

$$
\hat{\mathcal{H}}=\sum_{i}\left\{J_{1} \hat{\mathbf{S}}_{i} \cdot \hat{\mathbf{S}}_{i+1}+J_{2}\left[1+(-1)^{i} \delta\right] \hat{\mathbf{S}}_{i} \cdot \hat{\mathbf{S}}_{i+2}\right\}
$$

where $J_{1}, J_{2} \geq 0$ are the nearest- and next-nearest neighbor exchange couplings, respectively and $0 \leq \delta \leq 1$ is the bond alternation parameter. In the following, all the energies are expressed in units of $J_{1}$. For $\delta=0$ the properties of the model and the existence of a quantum phase transition at $\left(J_{2} / J_{1}\right)_{c} \simeq 0.241$ are well established ${ }^{7.8} \mathrm{Be}-$ low the critical point the system is in a Luttinger spinfluid phase with a gapless spectrum, no broken symmetry, and a finite spin-wave velocity. Spin correlations are characterized by a power-law decay defined by the same exponent of the pure Heisenberg chain $\left(J_{2}=0\right)$. In the regime of strong frustration, instead, the ground state is spontaneously dimerized, two-fold degenerate and adiabatically connected to the Majumdar-Gosh exact solution for $J_{2} / J_{1}=0.5^{9.10}$ with exponentially decaying spin correlations.

The effect of the bond alternation on the n.n.n. interaction has been recently investigated using fieldtheoretical approaches 1.2 with remarkable disagreement in the conclusions. In fact, Chen and collaborators 1 using bosonization and a renormalization group analysis claimed that in the limit of small frustration the asymmetry in the n.n.n. integrals destabilizes the isotropic
Heisenberg fixed point leading to a new phase with gapless excitations and vanishing spin-wave velocity. On the other hand, with a similar bosonization approach Sarkar and $\mathrm{Sen}^{2}$ found that bond alternation represents an irrelevant perturbation in the regime of small frustration. In this paper, using conformal field theory we show that the n.n.n. spin-Peierls operator, i.e., the one associated with the alternation in the n.n.n. exchange,

$$
\hat{O}_{n . n . n .}=\sum_{l}(-1)^{l} \hat{\mathbf{S}}_{l} \cdot \hat{\mathbf{S}}_{l+2},
$$

represents indeed an irrelevant perturbation for the Heisenberg chain in the regime of weak frustration. Furthermore, using Lanczos diagonalization technique we find that in the dimerized phase the effect of the bond alternation is to reduce the spin gap leading therefore to a slight enhancement with $\delta$ of the critical value $\left(J_{2} / J_{1}\right)_{c}$ for the fluid-dimer transition. Finally, we present an accurate numerical determination of the shift introduced by the bond alternation on the phase boundary between the Luttinger and the dimerized phase. A preliminary account of this work was already presented in Ref. 11.

In general, the relevance of the operator (2) for the Luttinger fixed point can be determined by calculating its scaling dimension $X$. This can be defined in terms of the size-dependence of its generalized susceptibility

$$
\chi_{O}=\frac{2}{L}\left\langle\psi_{0}\left|\hat{O}\left(E_{0}-\hat{\mathcal{H}}\right)^{-1} \hat{O}\right| \psi_{0}\right\rangle \propto L^{2(1-X)},
$$

where $L$ is the number of sites of the ring, so that $X<1$ characterizes a relevant operator $\frac{12}{1}$ Here $\left|\psi_{0}\right\rangle$ represents the unperturbed Luttinger ground state. The calculation of $X$ can be done by identifying the quantum numbers $\{j\}$ (referenced to the ground state with energy $E_{0}$ ) of the intermediate states $|j\rangle$ appearing in the Lehmann representation of $\chi_{O} \stackrel{12}{12}$ In fact, in a conformally invariant field theory, the scaling dimension $X$ of a given operator 
is related to the finite-size corrections of the energy of the lowest intermediate eigenstate $|j\rangle$ by

$$
\Delta E(L)=E_{j}(L)-E_{0}(L)=2 \pi v_{s} X / L,
$$

where $v_{s}$ is the spin-wave velocity. In the case of the n.n.n. operator (21), the quantum numbers of the intermediate states, referenced to the ground state, are the total spin $\hat{\mathbf{S}}=0$, momentum $k=\pi$ and even parity under spatial reflection $(l \rightarrow L-l), R=1$. In all cases, the only difference with the nearest-neighbor (n.n.) spinPeierls operator,

$$
\hat{O}_{n . n .}=\sum_{l}(-1)^{l} \hat{\mathbf{S}}_{l} \cdot \hat{\mathbf{S}}_{l+1},
$$

the well-known relevant perturbation, is the spatialreflection quantum number, $R=-1$. The finite-size corrections can be computed either by Bethe ansatz (for $\left.J_{2} / J_{1}=0\right)$ or by bosonization. According to the standard notation, at the Luttinger fixed point, the effective Hamiltonian describing spin fluctuations is given by a free boson theory $\stackrel{13}{\underline{13}}$

$$
H_{L}=\frac{v_{s}}{2} \int d x\left\{K \Pi^{2}(x)+\frac{1}{K}\left[\partial_{x} \Phi(x)\right]^{2}\right\},
$$

where $v_{s}$ is the spin velocity, $\Phi(x)$ is the spin fluctuation field and $\Pi(x)$ is the conjugated moment. The dimensionless coupling constant $K$ is the key parameter of the bosonization theory, whose value can be analytically determined at $J_{2}=0$ by comparing the low-lying excitation spectrum of the Hamiltonian (6) with the Bethe ansatz solution of the lattice model: $K=\left(2-2 \cos ^{-1} \lambda / \pi\right)^{-1}$. Here $0 \leq \lambda \leq 1$ is the coupling constant defining the easy-plane anisotropy: at the spin-isotropic point $\lambda=1$, leading to $K=1 / 2$. The finite-size corrections of the lowest excitation energies of this Hamiltonian are given by 14

$$
E_{n, m}(L)-E_{0}(L)=\frac{2 \pi v_{s}}{L}\left(\frac{n^{2}}{4 K}+K m^{2}\right) .
$$

For each pair of integers $n$ and $m(n=m=0$ being the ground state), a low-energy excited state with energy $E_{n, m}$ is defined by choosing appropriate boundary conditions for the fields:

$$
\begin{aligned}
\Phi(L)-\Phi(0) & =n \sqrt{\pi}, \\
\int_{0}^{L} d x \Pi(x) & =2 m \sqrt{\pi} .
\end{aligned}
$$

Using the relation between the boson fields and the physical electron operator ones, it was then derived in Ref. 13 that $n$ is the $z$ component of the spin, while $m+n$ is the momentum of the low-energy excitation in units of $\pi$. Following this work, it can be shown that the reflection symmetry $\mathcal{R}$ acts on the fields as follows:

$$
\begin{aligned}
& \mathcal{R}^{\dagger} \Phi(x) \mathcal{R}=-\Phi(L-x), \\
& \mathcal{R}^{\dagger} \Pi(x) \mathcal{R}=-\Pi(L-x),
\end{aligned}
$$

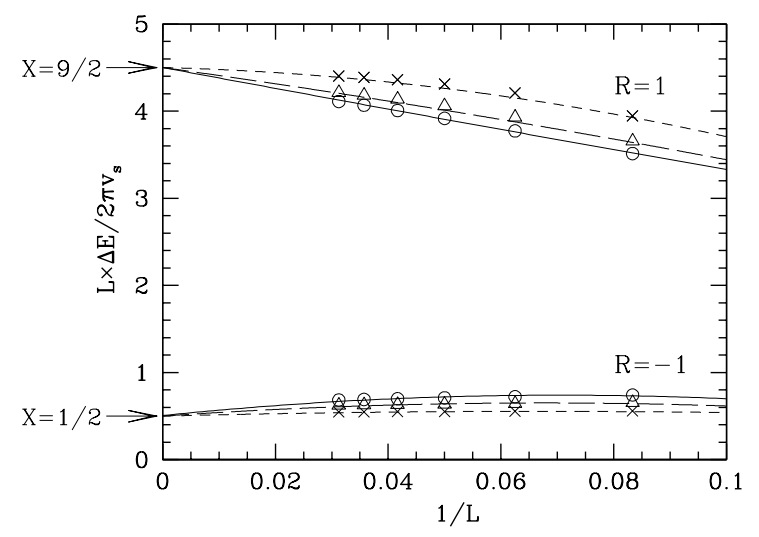

FIG. 1: Size scaling of the gap of the lowest eigenvalues of the isotropic spin-half $J_{1}-J_{2}$ chain with $S^{z}=0, k=\pi$, even parity under spin reflections, and $R= \pm 1$ under lattice reflections: $J_{2} / J_{1}=0$ (circles), 0.1 (triangles), and 0.2 (crosses). Lines are guides for the eye.

implying that the quantum number $m$ changes sign under spatial reflection $\mathcal{R}$, whereas $n$ does not, and, therefore, the ground state has $R=1$. For $m \neq 0$, each pair $\pm m$ leads to two degenerate eigenstates with opposite reflections, $R= \pm 1$.

Clearly, the excitations of Eq. (7) do not exhaust the full energy spectrum of the Luttinger model because, on top of them, the usual boson spectrum is also present:

$$
E_{k}(L)=v_{s} \sum_{k \neq 0}|k| a_{k}^{\dagger} a_{k}
$$

where $a_{k}^{\dagger}$ is a boson field defined in terms of $\Phi(x)$ and $\Pi(x)$, which satisfies $\mathcal{R}^{\dagger} a_{k}^{\dagger} \mathcal{R}=-a_{-k}^{\dagger}$.

For both the spin-Peierls operators previously defined, the appropriate choices of quantum numbers are $n=0$ (i.e., $S^{z}=0$ ) and $m=$ odd (i.e., momentum $\pi$ ). The two lowest excitations with $m= \pm 1$ and $n=0$ can be combined to construct two states with defined reflection parity, $R= \pm 1$. In the spin-isotropic case, $K=1 / 2$, the excitations with $(m= \pm 1, n=0)$ and $(m=0, n= \pm 1)$ are degenerate, the latter ones corresponding to a $R=1$ triplet (the spatial reflection does not change $n$ ). Therefore, the state with $n=0$ and $R=1$ is the $S^{z}=0$ state of the triplet, and the remaining state with $n=0$ and $R=-1$ has to be a singlet. Analogously, all finite- $m$ excitations with $n=0$ are forbidden to be singlets with $R=1$ by the spin isotropy. In order to obtain a lowenergy singlet excitation with $R=1$, we have to combine the $m=1(R=-1)$ excitation with at least three $a_{k}^{\dagger}$ bosons with zero total momentum. In fact one boson cannot have zero momentum and two bosons cannot change the spatial reflection symmetry. The minimum energy of such state is readily evaluated [with $k_{1}=k_{2}=2 \pi / L$, and $\left.k_{3}=-\left(k_{1}+k_{2}\right)\right]$ as

$$
\Delta E(L)=2 \pi v_{s}(K+4) / L,
$$




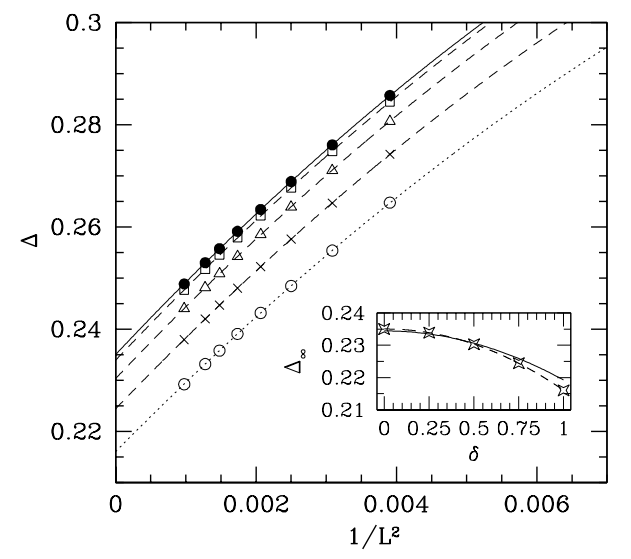

FIG. 2: Size scaling of the triplet gap at $J_{2} / J_{1}=0.5$ for $\delta=0$ (full circles), 0.25 (squares), 0.5 (triangles), 0.75 (crosses), and 1.0 (empty circles). Lines are quadratic fits. Inset: thermodynamic gap versus $\delta$ obtained by extrapolation (stars and dashed line) and with the variational approach of Refs. 1310 (continuous line).

leading to the result $X=K+4$ for any operator with the same symmetries of the operator (2).

Although the previous equation has been extracted in the isotropic case $(\lambda=1)$, where $K=1 / 2$, it is indeed valid in general for $0 \leq \lambda \leq 1$, as can be verified, analytically, in the XY model (i.e., at $\lambda=0$ ) or, numerically, for $\lambda<1$ (not shown). The reason is that, in the entire gapless regime, the low-energy excitations are described by Eqs. (77) and (12). Notice that, for $\lambda \neq 1$, the total spin is no longer a good quantum number, nevertheless the parity under spin-reflection $\left[\left(S^{x}, S^{y}, S^{z}\right) \rightarrow\left(-S^{x}, S^{y},-S^{z}\right)\right]$ can still be used to label the low-lying states.

The previous analysis shows that the finite-size spectrum of the Luttinger chain does not contain low-lying singlets with $k=\pi$ and even parity under spatialreflections, yielding to a critical exponent $X>1$ for the operator (2), and therefore proving its irrelevance. This is also illustrated in Fig. 1] where we report exact diagonalization results in the weak frustration regime of the isotropic chain. 15 However, we stress that our conclusions follow directly from the bosonization technique and represent an exact result for the isotropic spin-half Heisenberg chain.

The irrelevance of the n.n.n. spin-Peierls perturbation in the regime of weak frustration can be also confirmed numerically from the Lanczos calculation of the associated generalized susceptibility. As explained in Ref. 16, this can be done by adding to the Hamiltonian the considered perturbation $\eta \hat{O}$ and then computing the groundstate energy per site $e(\eta)$ for few values of $\eta$ in order to estimate numerically $\chi=-d^{2} e(\eta) /\left.d \eta^{2}\right|_{\eta=0}$ as the limit $\chi=\lim _{\eta \rightarrow 0} 2\left(e_{0}-e(\eta)\right) / \eta^{2}$. For instance, for the 28 -site chain at $J_{2} / J_{1}=0.1$, this procedure gives $\chi_{n n} \simeq 6.4$ and $\chi_{n n n} \simeq 10^{-6}$, for the n.n. and n.n.n spin-Peierls
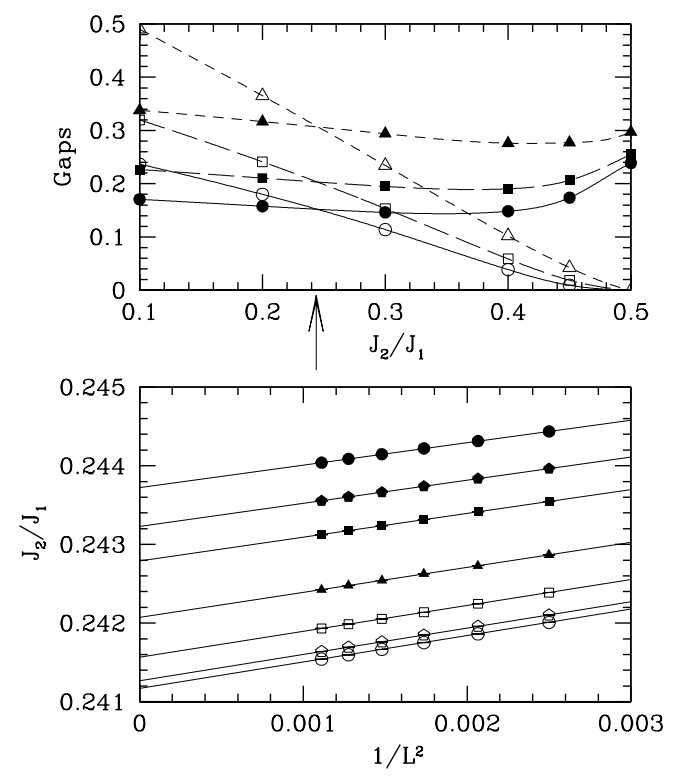

FIG. 3: Upper panel: singlet (open dots) and triplet (full dots) gaps versus $J_{2} / J_{1}$ for $\delta=1.0, L=12$ (triangles), 18 (squares), and 24 (circles). The arrow indicates the extrapolated value of $J_{2} / J_{1}$ at the level crossing. Lines are guides for the eye. Lower panel: size scaling of $J_{2} / J_{1}$ at the level crossing for (from below) $\delta=0,0.2,0.4,0.6,0.8,0.9,1.0$. Lines are quadratic fits.

operator, respectively. This fact indicates that the response of the system to a n.n.n. dimerization is more than six orders of magnitude smaller than the response to the standard n.n. dimerization.

Entering the regime of strong frustration, the effect of the n.n.n. alternation is to reduce the spin gap $\Delta$ as it is shown in Fig. 2 at $J_{2} / J_{1}=0.5$. As it has been already pointed out 1.2 at the Majumdar-Gosh point $\left(J_{2} / J_{1}=0.5\right)$ the presence of the n.n.n. spin-Peierls dimerization does not alter the ground-state manifold which is spanned by the two well-known dimerized states, with energy $E_{0}=-3 / 8 N$, independent of $\delta$. Nevertheless, the excited states are not known exactly so that we have calculated the spin gap using the Lanczos technique. By extrapolating the finite-size data according to the expected law, $\Delta(L)=\Delta_{\infty}+a / L^{2}+b / L^{4}$, we have obtained the $\delta$ dependence of the thermodynamic gap, which is shown in the same figure. Our results are in good agreement with the variational calculation of Refs. 1 3 10, and indicate a $8 \%$ spin-gap reduction from the Majumdar-Gosh model for $\delta=0(\Delta=0.235)$ to the so-called saw-tooth chain for $\delta=1(\Delta=0.216), 18$

The reduction of the spin gap in the dimerized phase induced by the n.n.n. bond alternation leads to an increase of the critical value $\left(J_{2} / J_{1}\right)_{c}$ for the fluiddimer transition. As originally suggested by Castilla and collaborators, 19 the phase boundary between the two 


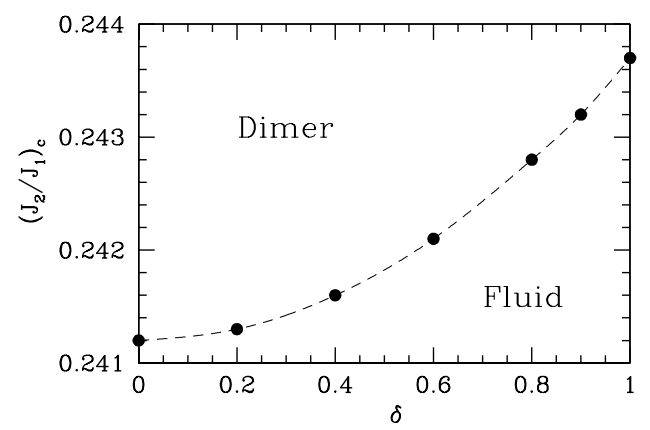

FIG. 4: Critical $J_{2} / J_{1}$ ratio for the fluid-dimer transition versus $\delta$.

phases can be calculated very accurately by exploiting the change in the excitation spectrum occurring at the critical point. In fact, below the critical point the system displays quasi-long-range antiferromagnetic order: in this case, gapless excitations are obtained by creating lowenergy spinons both in triplet (spatially even) and singlet (spatially odd) states, the former having the lowest energy on finite size. In the dimerized phase, instead, the triplet excitations acquire a gap so that the ground state remains two-fold degenerate in the singlet sector, leading to the dimerized ground state which breaks the translational invariance. As a result, the critical coupling $\left(J_{2} / J_{1}\right)_{c}$ can be determined by performing a size scaling of the value $J_{2} / J_{1}$ where the level crossing between the low-lying singlet and triplet occurs (see Fig. 3). With this technique, using exact diagonalizations up to 30 sites, it is easy to obtain very accurate determinations of the critical value $\left(J_{2} / J_{1}\right)_{c}$. For instance, in absence of n.n.n. bond alternation, we obtain a critical value $\left(J_{2} / J_{1}\right)_{c}$ in agreement with previously given estimates ${ }^{8.19}$ The resulting phase boundary for the fluid-dimer transition is

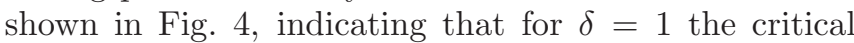
value of the frustration ratio for the fluid-dimer transition is only increased by about $1 \%$.

In conclusion, we have studied the effects of a nextnearest-neighbor spin-Peierls dimerization on the ground state of the spin-half $J_{1}-J_{2}$ Heisenberg chain, using conformal field theory and exact diagonalization. We have found that, despite the conclusions of a recent study based on bosonization and renormalization group approaches $\underline{\underline{1}}$ the bond alternation represents an irrelevant perturbation in both the regime of strong and weak frustration. In particular, as predicted by Sarkar and Sen,$^{2}$ the perturbation does not change the nature of the excitation spectrum, leading only to a weak reduction of the triplet gap in the dimerized phase, and, therefore, to a slight enhancement of the critical value $\left(J_{2} / J_{1}\right)_{c}$ associated to the fluid-dimer transition.

We would like to thank D. Sen for having attracted our attention to this model and for useful correspondence. L.C. and A.P. acknowledge kind hospitality at SISSA. This work was partially supported by MIUR (COFIN01) and INFM (PAIS-MALODI). L.C. was supported by NSF grant DMR-9817242.
1 S. Chen, H. Büttner, and J. Voit, Phys. Rev. Lett. 87, 087205 (2001); ibidem 89, 149702 (2002); e. print cond-mat/0201004

2 S. Sarkar and D. Sen, Phys. Rev. B 65, 172408 (2002).

3 D. Sen, S. Shastry, R.E. Walstedt, and R. Cava, Phys. Rev. B 53, 6401 (1996).

4 S.A. Blundell and D.M. Núñez-Regueiro, e. print cond-mat/0204405

5 T. Nakamura and K. Kubo, Phys. Rev. B 53, 6393 (1996); T. Nakamura and S. Takada, Phys. Rev. B 55, 14413 (1997).

${ }^{6}$ G. Van Tendeloo, O. Garlea, C. Darie, C. BougerolChaillout, and P. Bordet, J. Solid State Chem. 156, 428 (2001).

7 S.R. White and I. Affleck, Phys. Rev. B 54, 9862 (1996).

8 S. Eggert, Phys. Rev. B 54, R9612 (1996).

9 C.K. Majumdar and D.K. Gosh, J. Math. Phys. 10, 1388, (1969); ibidem 10, 1399 (1969)

10 B. S. Shastry and B. Sutherland, Phys. Rev. Lett. 47, 964 (1981).

11 L. Capriotti, F. Becca, S. Sorella, and A. Parola, Phys. Rev. Lett. 89, 149701 (2002).
12 J.L. Cardy, Nucl. Phys. B270 [FS16], 186 (1986).

13 S. Eggert and I. Affleck Phys. Rev. B 46, 10866 (1992); I. Affleck, in Field Strings and Critical Phenomena, edited by E. Brezin and J. Zinn-Justin (North-Holland, Amsterdam, 1989).

14 S. Sorella and A. Parola, Phys. Rev. B 57, 6444 (1998).

15 The spin-wave velocity $v_{s}$ is known exactly only for $J_{2}=0$, where $v_{s}=\pi / 2$. However, in the Luttinger regime it can be obtained numerically from the finite-size corrections to the ground-state energy according to the relation, $E_{0}(L)=$ $e_{0} L-v_{s} \pi / 6 L$, where $e_{0}$ is the thermodynamic groundstate energy per site. See for instance, K. Nomura and K. Okamoto, J. Phys. A 27, 5773 (1994).

16 L. Capriotti and S. Sorella, Phys. Rev. Lett. 84, 3173 (2000).

17 S.R. White, R.M. Noack, and D.J. Scalapino, Phys. Rev. Lett. 73, 886 (1994).

18 The value for the saw-tooth chain $(\delta=1)$ is in agreement with the recent calculation of Ref. 4 .

19 G. Castilla, S. Chakravarty, and V.J. Emery, Phys. Rev. Lett. 75, 631 (1995). 